\title{
Effects of Meat Intake Frequency and Polymorphic Cytochrome P450 2A6 Activity on Individual Colorectal Tumour Risk in a Japanese Cohort
}

\author{
Hiroshi Yamazaki1 ${ }^{*}$, Masaki Fujieda², Makiko Shimizu', Akiko Shiotani' ${ }^{3}$, Mie Shimabukuro4, \\ Kanae Mure ${ }^{4}$, Tatsuya Takeshita ${ }^{4}$, Hideki Ishikawa ${ }^{5 *}$
}

\author{
${ }^{1}$ Laboratory of Drug Metabolism and Pharmacokinetics, Showa Pharmaceutical University, Machida, Tokyo, Japan \\ ${ }^{2}$ Faculty of Pharmaceutical Sciences, Toho University, Funabashi, Japan \\ ${ }^{3}$ Otemae College of Nutrition and Confectionery, Chuo-ku, Osaka, Japan \\ ${ }^{4}$ Department of Public Health, Wakayama Medical University, Wakayama, Japan \\ ${ }^{5}$ Department of Molecular-Targeting Cancer Prevention, Kyoto Prefectural University, Sakyo-ku, Kyoto, Japan \\ Email: ^hyamazak@ac.shoyaku.ac.jp.orcid.org, *cancer@gol.com
}

How to cite this paper: Yamazaki, H., Fujieda, M., Shimizu, M., Shiotani, A., Shimabukuro, M., Mure, K., Takeshita, T. and Ishikawa, H. (2017) Effects of Meat Intake Frequency and Polymorphic Cytochrome P450 2A6 Activity on Individual Colorectal Tumour Risk in a Japanese Cohort. Journal of Cancer Therapy, 8, 645-652.

https://doi.org/10.4236/jct.2017.87055

Received: June 13, 2017

Accepted: July 15, 2017

Published: July 18, 2017

Copyright $\odot 2017$ by authors and Scientific Research Publishing Inc. This work is licensed under the Creative Commons Attribution International License (CC BY 4.0).

http://creativecommons.org/licenses/by/4.0/

\begin{abstract}
The relationships among meat consumption, smoking habits, and phenotypic cytochrome P450 2A6 variation with respect to colorectal cancer risk remain unclear. In this study, the relationships among colorectal tumour risk, meat consumption by questionnaire, and phenotypic P450 2A6 activity by genotyping in a case-control study (299 cases and 170 controls) were analyzed for never-smokers and ever-smokers. In never-smokers consuming $\geq 1$ serving per day of total meat, a significant odds ratio of 4.42 (95\% confidence interval, 1.29 - 15.2), adjusted by logistic regression for age and gender, was observed, compared with the group consuming $\leq 2$ servings per week. Furthermore, in Japanese never-smokers, the susceptibility to colorectal tumours was dependent on the frequency of meat intake (trend test $p=0.011$ ). In never-smokers who were P450 2A6 poor metabolizers and had a high frequency of meat intake, the apparent odds ratio was 3.57 (95\% confidence interval, 0.30 - 42.2) compared with the P450 2A6 normal group with a low meat intake frequency. These results suggested that colorectal tumour risk was inversely associated with the phenotypic P450 2A6 activities in Japanese never-smokers with a high meat intake.
\end{abstract}

\section{Keywords}

Meat Consumption, Colon Cancer Risk, Susceptibility, Non-Smoking Subjects, CYP2A6 


\section{Introduction}

The consumptions of red meat, processed meat, and high-fat products and smoking have been positively associated with colorectal tumour risk in previous epidemical studies [1] [2] [3] [4] [5]. Epidemiological and human biomonitoring studies indicate that heterocyclic amines, polycyclic hydrocarbons, and $\mathrm{N}$-nitrosamines in meat-derived products may play important roles in colorectal carcinogenesis [6] [7].

Cigarette smoking has been consistently identified as a potential risk factor for colorectal tumours [2] [8]. Because polymorphic cytochrome P450 (P450) 2A6 enzymes mediate nicotine oxidation and the metabolic activation of several procarcinogens, including environmental and tobacco-related $N$-nitrosamines [9] [10], the involvement of P450 2A6 in cancer development has been postulated. However, there have been contradictory findings about the role of P450 2A6 in colorectal carcinogenesis. In a case-control study, subjects with medium or high P450 2A6 activities (as determined by the urinary caffeine test) were found to have an increased risk of colorectal cancer compared with subjects with low P450 2A6 activity [6]. In contrast, higher P450 2A6 activities were found not to be associated with the colorectal cancer risk in a different study [11]. We previously found that a high risk of colorectal tumours in Japanese subjects was associated with higher P450 2A6 activity and higher cumulative tobacco exposure [8]. Moreover, the susceptibility to colorectal tumours was dependent on the predicted P450 2A6 phenotype among Japanese ever-smokers, but not among never-smokers [8]. However, compared with the abundant data from Western countries [1] [2] [3] [4], evidence regarding meat consumption and colorectal cancer is limited in the Japanese population. Furthermore, the Japanese population is known to have a wide range of $\mathrm{P} 450$ phenotypes with different enzyme activities [12]. The relationships among meat consumption, smoking habits, and phenotypic P450 2A6 variation with respect to colorectal cancer risk remain unclear.

In the current study, we found that the susceptibility to colorectal tumours was dependent on the frequency of meat intake in Japanese never-smokers. Moreover, on reanalysis of our previous case-control study, the risk for colorectal tumours was found to be inversely dependent on the enzyme activity of the predicted P450 2A6 phenotype among Japanese never-smokers with a high meat intake.

\section{Materials and Methods}

\subsection{Subjects}

Study participants recruited from June 1993 to September 1997 at the Osaka Medical Center for Cancer and Cardiovascular Diseases [8] were reanalyzed in this study. The colorectal tumour cases included adenomas and adenocarcinomas ( $\mathrm{n}=299,81 \%$ male) but excluded individuals with a history of intestinal or gastric resection, familial adenomatous polyposis, or advanced or invasive tu- 
mours. Control subjects were healthy volunteers $(n=170,79 \%$ male) who required hospitalization for a health check-up. The ages of the cases (56 years) and the controls ( 58 years) were defined as those at the time of the first hospital visit. Signed consent forms and completed questionnaires were collected from all subjects. The questionnaire covered smoking status (never-smoker, ex-smoker, or current smoker), the total duration of smoking, the average number of cigarettes smoked daily, and meat intake ( $\leq 2$ serving per week, 1 serving per 2 days, or $\geq 1$ serving per day) as shown in Table 1 . The number of pack-years was calculated as a measure of cumulative cigarette smoking: one pack-year was defined as smoking 20 cigarettes daily for 1 year. Meat was defined as beef, pork, poultry, ham, and sausages. This study was approved by the ethics committees of Kyoto Medical University and Showa Pharmaceutical University.

\subsection{Data Collection and Analysis}

The genotyping of $P 4502 A \sigma^{\star} 4$ (whole-gene deletion), $2 A \sigma^{\star} 7$ (amino acid substitution), and $2 A \sigma^{*} 9$ (upstream mutation) were performed using a previously described method [8] [12] [13]. Subjects were assigned to four groups based on their P450 $2 A 6$ genotypes according to the definitions of Fujieda et al. [12]: the putative normal phenotypic group, $\left.2 A \sigma^{\star} 1\right|^{\star} 1$; the intermediate group, i.e., those heterozygous for the $P 4502 A 6^{\star} 1$ allele $\left(2 A 6^{\star} 1{ }^{\star} 4,{ }^{*} 7,{ }^{*} 9\right)$; the slow group, i.e., those heterozygous or homozygous for variant alleles except for those homozygous for the $P 4502 A \sigma^{\star} 4$ allele $\left(2 A 6^{*} 4,{ }^{*} 7,\left.{ }^{*}\right|^{*} 7,{ }^{*}\right)$ ); and the poor group, $P 450$ $\left.2 A \sigma^{*} 4\right|^{*} 4$. Associations between susceptibility to colorectal tumours and smoking history, meat intake, and phenotypic P450 2A6 activity were assessed using odds ratios and 95\% confidence intervals calculated from logistic regression models with adjustment for age and gender and/or smoking pack-years (in smokers). $p$ values for trends were also calculated by assigning ordinal scores as continuous variables in the logistic regression models, with the ordinal scores 1 3 assigned to the three levels of meat intake or ordinal scores of $1-4$ assigned to the normal P450 2A6 phenotype and intermediate, slow, and poor metabolizer groups, respectively [8]. All tests of statistical significance were two-sided. A $p$

Table 1. The numbers of participants in this study.

\begin{tabular}{ccccc}
\hline & \multicolumn{4}{c}{ Number of subjects } \\
\cline { 2 - 4 } Frequency of meat intake & \multicolumn{2}{c}{ Never-smokers } & \multicolumn{2}{c}{ Ever-smokers } \\
\cline { 2 - 4 } & Case & Control & Case & Control \\
\hline Low frequency & 34 & 49 & 108 & 48 \\
$\leq 2$ serving per week & & & & 25 \\
Medium/high frequency & 37 & 30 & 80 & 14 \\
1 serving per 2 days & 12 & 4 & 28 & 25 \\
\hline 1 serving per day & & & & 48 \\
\hline
\end{tabular}

The colorectal tumour cases included adenomas and adenocarcinomas ( $\mathrm{n}=299,81 \%$ male). Control subjects were healthy volunteers $(n=170,79 \%$ male). The mean ages of the cases and controls were 56 and 58 years, respectively. 
value of 0.05 was considered the threshold of significance. All statistical analyses were carried out by using the statistical software SAS, version 5.0 (SAS Institute, Inc., Cary, NC, USA).

\section{Results}

The frequency of meat intake was analyzed in a case-control study of colorectal tumours in a Japanese cohort divided into ever-smokers (i.e., ex-smokers and current smokers) and never-smokers. For those with a total meat intake of $\geq 1$ serving per day compared with $\leq 2$ servings per week, the odds ratio for the presence of colorectal tumours adjusted by logistic regression for age, gender, and smoking pack-years was 1.48 (95\% confidence interval, $0.78-2.81$ ); however, this result was not statistically significant (data not shown). Because the highest risk of colorectal tumours was previously found in subjects with both high P450 2A6 activity and high cumulative tobacco exposure [8], separate analyses were performed for never-smokers and ever-smokers (Figure 1). In never-smokers with a high total meat intake of $\geq 1$ serving per day (12 cases and 4 controls, Figure 1(a)) compared with $\leq 2$ servings per week (34 cases and 49 controls), we observed a significant odds ratio of 4.42 ( $95 \%$ confidence interval, 1.29 - 15.2, Figure 1(c)) adjusted by logistic regression for age and gender. Furthermore, with ordinal scores of $1-3$ assigned to the low ( $\leq 2$ servings per week), medium ( 1 serving per 2 days), and high ( $\geq 1$ serving per day) frequencies of meat intake, in Japanese never-smokers, the susceptibility to colorectal tumours was found to be dependent on the frequency of meat intake (trend test $p=0.011$, Figure 1(c)). Moreover, in ever-smokers with low, medium, and high meat intake (Figure 1(b)), respectively, significant and similar odds ratios of 4.09 (95\% confidence interval, 2.19 - 7.66), 5.48 (2.75 - 10.9), and 3.46 (1.50 - 7.98), adjusted by logistic regression for age, gender, and smoking pack-years, were observed

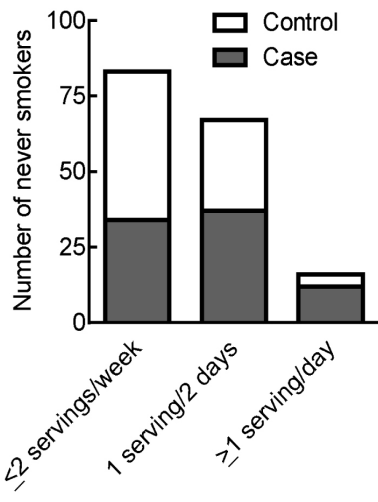

(a)

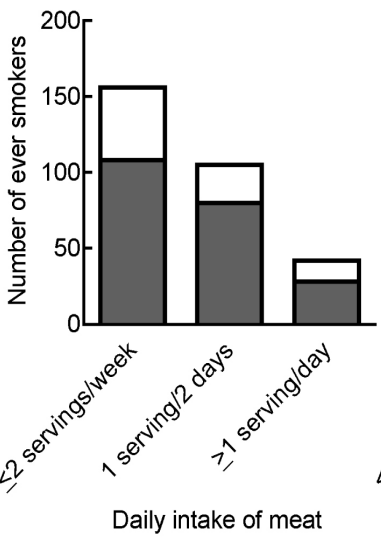

(b)

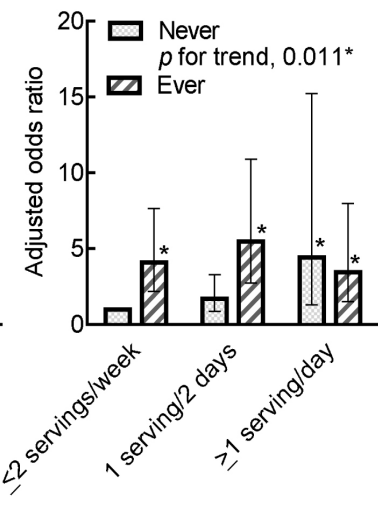

(c)

Figure 1. Frequency of meat intake and risk of colorectal tumours in never-smokers (a) and ever-smokers (b) in a case-control study. Odds ratios with $95 \%$ confidential interval values (c) adjusted for age and gender are shown compared with never-smokers with a low meat intake as the reference group. $p$ values for trends (c) were calculated by assigning ordinal scores as continuous variables in the logistic regression models with the ordinal scores 1 - 3 assigned to the three frequencies of meat intake. 
compared with never-smokers with low meat intake (Figure 1(c)).

Associations between susceptibility to colorectal tumours, frequency of meat intake, and putative P450 2A6 activity based on the P450 2A6 genotype were assessed in never-smokers (Figure 2). In the low meat intake group, no significant odds ratios or trends were evident for the different P450 2A6 genotypes. However, in the combined medium and high meat intake groups (Figure 2(b)), poor metabolizers ( 3 cases and 1 control) had an apparent odds ratio of 3.57 (95\% confidence interval, 0.30 - 42.2, Figure 2(c)) adjusted by logistic regression for age and gender compared with the low meat intake group with the normal P450 2A6 phenotype (8 cases and 10 controls, Figure 2(c)). In Japanese never-smokers, the risk for colorectal tumours was suggested to be inversely associated with the predicted activities of the P450 2A6 phenotypes in the combined medium and high meat intake groups (trend test $p=0.045$, Figure $2(\mathrm{c})$ ).

\section{Discussion}

There is accumulating evidence that both diet and smoking are major environmental risk factors for colorectal tumours [1] [2] [3] [5]. The activities of polymorphic enzymes (glutathione $S$-transferase A1, sulfotransferase $1 \mathrm{~A} 1$, and $N$ acetyltransferase 2) associated with the metabolism of heterocyclic amines and polycyclic hydrocarbons, were found to be possibly associated with colon cancer risk [3]. Furthermore, in a population-based prospective cohort study, the intake of red and processed meat reportedly increased the risk of colorectal or colon cancer among Japanese men, but not among Japanese women [5]. In the current study, the effects of the frequency of meat intake and smoking habits (acquired by questionnaires) were obtained by reanalysis of our previous case-control study of colorectal tumours [8]. The roles of phenotypic P450 2A6 activity were assessed, especially in subjects who had never smoked. In never-smokers, the

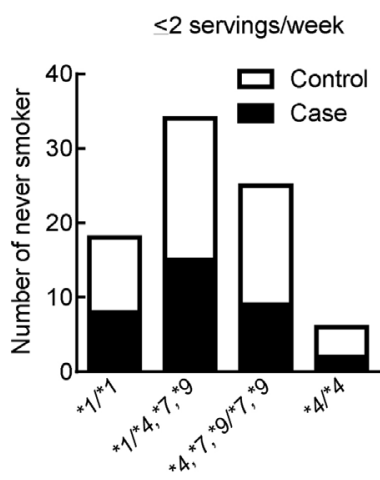

(a)

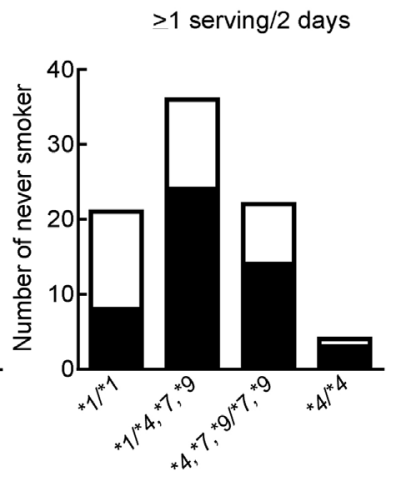

P450 2A6 genotype

(b)

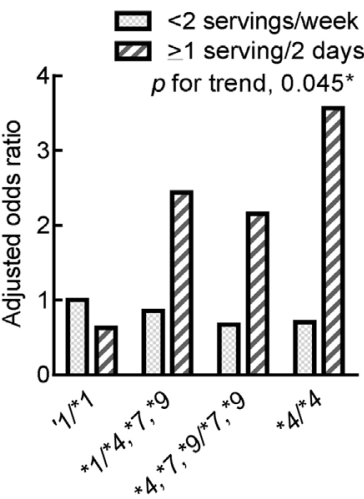

(c)

Figure 2. Effects of phenotypic P450 2A6 activity and low (a) and medium/high (b) frequency of meat intake on the risk of colorectal tumours in never-smokers in a case-control study. A low frequency of meat intake was defined as $\leq 2$ servings per week and a high frequency of meat intake was defined as $\geq 1$ serving per 2 days. Odds ratios (c) adjusted for age and gender are shown compared to never-smokers with a low intake of meat with normal P450 2 A6 activity ( $P 4502 A 6^{\star} 1{ }^{\star} 1$ genotype) as the reference group. 
high meat intake group had a significant adjusted odds ratio of 4.42 compared with the low meat intake group (Figure 1). The susceptibility to colorectal tumours was significantly dependent on the frequency of meat intake in Japanese never-smokers (Figure 1).

Subjects with the high P450 2A6 activity phenotype reportedly either have an increased risk [6] or no increased risk [5] of colorectal cancer. In Asian populations, such as the Japanese population, a relatively high frequency of P450 2A6 whole-gene deletion has been identified [12], which is different from that in Western populations. In the current study, never-smokers in the combined medium and high meat intake groups who were P450 2A6 poor metabolizers had an apparent adjusted odds ratio of 3.57 compared with low-meat-intake neversmokers with the normal P450 2A6 phenotype (Figure 2). In terms of the individual colorectal cancer risk of high-meat-intake never-smokers who were P450 $2 \mathrm{~A} 6$ poor metabolizers (Figure 2), our assessment suggested that dietary procarcinogens or undefined compounds may be predominantly deactivated by polymorphic P450 2A6 and, consequently, poor metabolizers would be at increased risk of colorectal cancer. To our knowledge, these causal factor(s) that are deactivated by P450 $2 \mathrm{~A} 6$ remain to be elucidated. We previously found the highest risk of colorectal tumours to be in Japanese subjects with high P450 2A6 activity and high cumulative tobacco exposure [8]. In contrast, here we suggest that the potential colorectal cancer risk in those with a medium or high meat intake is inversely associated with polymorphic P450 2A6 activity in a Japanese cohort, especially in those who have never smoked.

Our results are consistent with epidemiological studies of colorectal tumours that observed an increased risk with the frequency of meat intake. The inverse association of colorectal cancer risk with phenotypic P450 2A6 activity identified in the trend analysis $(p=0.045)$ of never smokers with high meat intake may somewhat attenuate the smoking risk of colorectal tumours in those with normal P450 2A6 activity in a Japanese cohort. Additional studies with detailed assessments of meat intake are needed to further evaluate the contributions of various components of meat intake, smoking habit, and other lifestyle factors to the risk of colorectal tumours in a cohort genotyped for P450 2A6. In conclusion, colorectal tumour risk was inversely associated with the phenotypic P450 2A6 activities in Japanese never-smokers with a high meat intake.

\section{Acknowledgements}

The authors thank Asami Muroi, Kazuma Kiyotani, and Tetsuya Kamataki for their assistance and David Smallbones for his advice on English language usage.

\section{Sources of Funding}

This study was supported in part by the Practical Research for Innovative Cancer Control from Japan Agency for Medical Research and Development, AMED (17ck0106276h0001). 


\section{Declaration of Interest}

The authors report no declarations of interest.

\section{References}

[1] Sandhu, M.S., White, I.R. and McPherson, K. (2001) Systematic Review of the Prospective Cohort Studies on Meat Consumption and Colorectal Cancer Risk: A Meta-Analytical Approach. Cancer Epidemiology, Biomarkers \& Prevention, 10, 439446.

[2] Giovannucci, E. (2001) An Updated Review of the Epidemiological Evidence that Cigarette Smoking Increases Risk of Colorectal Cancer. Cancer Epidemiology, Biomarkers \& Prevention, 10, 725-731.

[3] Nowell, S., Coles, B., Sinha, R., MacLeod, S., Luke, R.D., Stotts, C., Kadlubar, F.F., Ambrosone, C.B. and Lang, N.P. (2002) Analysis of Total Meat Intake and Exposure to Individual Heterocyclic Amines in a Case-Control Study of Colorectal Cancer: Contribution of Metabolic Variation to Risk. Mutation Research/Fundamental and Molecular Mechanisms of Mutagenesis, 506-507, 175-185.

https://doi.org/10.1016/S0027-5107(02)00164-1

[4] Ferrucci, L.M., Sinha, R., Huang, W.Y., Berndt, S.I., Katki, H.A., Schoen, R.E., Hayes, R.B. and Cross, A.J. (2012) Meat Consumption and the Risk of Incident Distal Colon and Rectal Adenoma. British Journal of Cancer, 106, 608-616. https://doi.org/10.1038/bjc.2011.549

[5] Wada, K., Oba, S., Tsuji, M., Tamura, T., Konishi, K., Goto, Y., Mizuta, F., Koda, S., Hori, A., Tanabashi, S., Matsushita, S., Tokimitsu, N. and Nagata, C. (2017) Meat Consumption and Colorectal Cancer Risk in Japan: The Takayama Study. Cancer Science, 108, 1065-1070. https://doi.org/10.1111/cas.13217

[6] Nowell, S., Sweeney, C., Hammons, G., Kadlubar, F.F. and Lang, N.P. (2002) CYP2A6 Activity Determined by Caffeine Phenotyping: Association with Colorectal Cancer Risk. Cancer Epidemiology, Biomarkers \& Prevention, 11, 377-383.

[7] Wu, K., Giovannucci, E., Byrne, C., Platz, E.A., Fuchs, C., Willett, W.C. and Sinha, R. (2006) Meat Mutagens and Risk of Distal Colon Adenoma in a Cohort of U.S. Men. Cancer Epidemiology, Biomarkers \& Prevention, 15, 1120-1125. https://doi.org/10.1158/1055-9965.EPI-05-0782

[8] Muroi, A., Kiyotani, K., Fujieda, M., Ishikawa, H., Takeshita, T., Iwano, S., Yamazaki, H. and Kamataki, T. (2012) Effect of Genetic Polymorphism of CYP2A6 on Individual Susceptibility to Colorectal Tumors in Japanese Smokers. Journal of Cancer Therapy, 3, 207-215. https://doi.org/10.4236/jct.2012.34030

[9] Yamazaki, H., Inui, Y., Yun, C.H., Guengerich, F.P. and Shimada, T. (1992) Cytochrome P450 2E1 and 2A6 Enzymes as Major Catalysts for Metabolic Activation of $\mathrm{N}$-Nitrosodialkylamines and Tobacco-Related Nitrosamines in Human Liver Microsomes. Carcinogenesis, 13, 1789-1794. https://doi.org/10.1093/carcin/13.10.1789

[10] Shimada, T., Kakimoto, K., Takenaka, S., Koga, N., Uehara, S., Murayama, N., Yamazaki, H., Kim, D., Guengerich, F.P. and Komori, M. (2016) Roles of Human CYP2A6 and Monkey CYP2A24 and 2A26 Cytochrome P450 Enzymes in the Oxidation of 2,5,2',5'-Tetrachlorobiphenyl. Drug Metabolism and Disposition, 44, 1899-1909. https://doi.org/10.1124/dmd.116.072991

[11] Ward, M.H., Cross, A.J., Divan, H., Kulldorff, M., Nowell-Kadlubar, S., Kadlubar, F.F. and Sinha, R. (2007) Processed Meat Intake, CYP2A6 Activity and Risk of Colorectal Adenoma. Carcinogenesis, 28, 1210-1216. https://doi.org/10.1093/carcin/bgm009 
[12] Fujieda, M., Yamazaki, H., Saito, T., Kiyotani, K., Gyamfi, M.A., Sakurai, M., Dosaka-Akita, H., Sawamura, Y., Yokota, J., Kunitoh, H. and Kamataki, T. (2004) Evaluation of CYP2A6 Genetic Polymorphisms as Determinants of Smoking Behavior and Tobacco-Related Lung Cancer Risk in MALE JAPANESE SMokers. Carcinogenesis, 25, 2451-2458. https://doi.org/10.1093/carcin/bgh258

[13] Murayama, N., Shimizu, M., Kobayashi, K., Kishimoto, I. and Yamazaki, H. (2016) Cytochrome P450 2A6 Phenotyping Using Dietary Caffeine Salivary Metabolite Ratios and Genotyping Using Blood on Storage Cards in Non-Smoking Japanese Volunteers. Drug Metabolism Letters, 10, 240-243.

https://doi.org/10.2174/1872312810666161114144008

\section{Scientific Research Publishing}

Submit or recommend next manuscript to SCIRP and we will provide best service for you:

Accepting pre-submission inquiries through Email, Facebook, LinkedIn, Twitter, etc. A wide selection of journals (inclusive of 9 subjects, more than 200 journals)

Providing 24-hour high-quality service

User-friendly online submission system

Fair and swift peer-review system

Efficient typesetting and proofreading procedure

Display of the result of downloads and visits, as well as the number of cited articles Maximum dissemination of your research work

Submit your manuscript at: http://papersubmission.scirp.org/

Orcontact jct@scirp.org 